\title{
Reengineering Aircraft Structural Life Prediction Using a Digital Twin
}

\author{
Eric J. Tuegel, ${ }^{1}$ Anthony R. Ingraffea, ${ }^{2}$ Thomas G. Eason, ${ }^{1}$ and S. Michael Spottswood \\ ${ }^{1}$ Structural Sciences Center, Air Vehicles Directorate, U.S. Air Force Research Laboratory, Wright-Patterson Air Force Base, \\ Dayton, $\mathrm{OH} 45433$, USA \\ ${ }^{2}$ School of Civil and Environmental Engineering, Cornell University, Ithaca, NY 14853, USA
}

Correspondence should be addressed to Eric J. Tuegel, eric.tuegel@wpafb.af.mil

Received 25 April 2011; Accepted 2 August 2011

Academic Editor: Nicholas Bellinger

Copyright (c) 2011 Eric J. Tuegel et al. This is an open access article distributed under the Creative Commons Attribution License, which permits unrestricted use, distribution, and reproduction in any medium, provided the original work is properly cited.

\begin{abstract}
Reengineering of the aircraft structural life prediction process to fully exploit advances in very high performance digital computing is proposed. The proposed process utilizes an ultrahigh fidelity model of individual aircraft by tail number, a Digital Twin, to integrate computation of structural deflections and temperatures in response to flight conditions, with resulting local damage and material state evolution. A conceptual model of how the Digital Twin can be used for predicting the life of aircraft structure and assuring its structural integrity is presented. The technical challenges to developing and deploying a Digital Twin are discussed in detail.
\end{abstract}

\section{Introduction}

Despite increasing capability to understand relevant physical phenomena and to automate numerical modeling of them, the process for lifing aircraft structure as outlined in Figure 1 has not advanced greatly in fifty years. The external loads on an aircraft (aerodynamic pressures and ground loads) are developed by the loads group using a specialized model and placed in a database. The loads for selected design points are pulled from the database by the structural modeling group who then apply them to the structural finite element model (FEM) to develop the internal loads in the airframe for each design load case. These cases are placed in a second database. The durability and damage tolerance experts use these internal load cases to develop stress transfer functions relating the external loads to local stresses at details such as fastener holes, cutouts, and fillets. The stress transfer functions are applied to the loads in the flight loads database to develop a stress spectrum at each point of interest in the airframe. These stress spectra are used in specialized fatigue software together with an idealized local geometry to predict the fatigue crack nucleation or fatigue crack growth lives of details that have been identified as fatigue sensitive. Meanwhile, the dynamics group uses yet another specialized model to determine the vibration characteristics of the aircraft to address the fatigue of the structure due to low-amplitude, high-frequency dynamic loads such as acoustic and aeroelastic.

Increased computational horsepower has enabled each of the individual parts of this process to be performed more efficiently, and so more load cases and fatigue locations can be analyzed. The output files from one model are more readily translated into input files for the next step of the process. However, there has been little effort made to integrate the physics in these individual models into a single comprehensive representation of the aircraft. Nor has the fidelity in the models of the physics increased significantly. The structural FEM is still a linear elastic model. The fatigue life prediction models are essentially the same ones that were used when the calculations were performed on handheld calculators. As a result, the identification of fatigue sensitive or other damage prone locations relies primarily on engineering judgment and fatigue tests. Because of judgment born of experience, evolution of existing aircraft designs is fairly successful; however, the development of revolutionary 


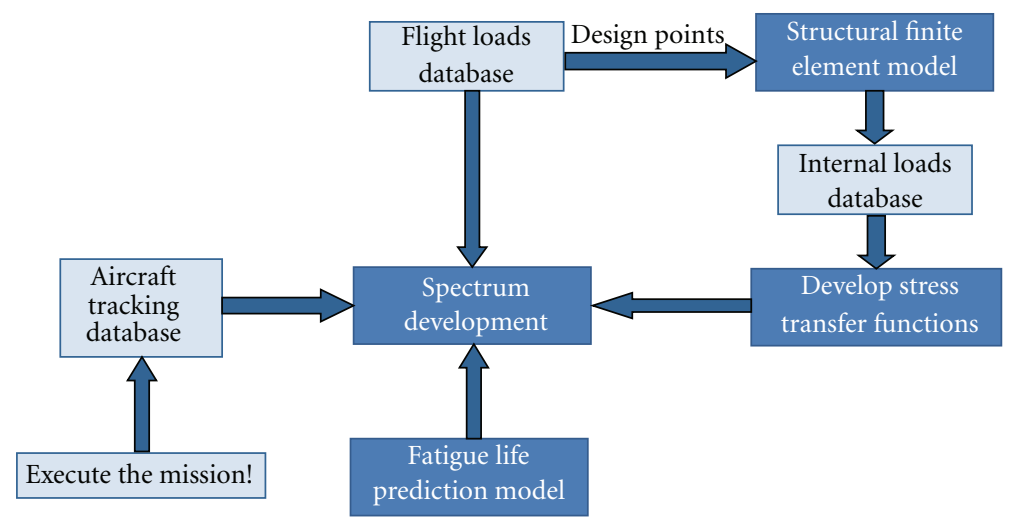

FIGURE 1: Schematic of current life prediction process.

aircraft is fraught with unexpected problems that result in weight growth, schedule delays, and cost overruns.

The life prediction process depicted in Figure 1 was the best that could be done in the years before digital computing was commonplace. The process was used to design the airframe to meet the design service life requirement and to establish the intervals at which to inspect locations on the airframe for fatigue cracks. The coming of the computer age led to the automation of various parts of this traditional life prediction process. However, the entire aircraft life prediction process has not been reexamined to see how the entire process can be reengineered as a result of the availability of high performance computing.

This paper presents a proposal for reengineering the structural life prediction process, hereafter called the Digital Twin, that utilizes the growing very high performance computational infrastructure. The Digital Twin concept developed from discussions the primary author had with T. A. Cruse, Professor Emeritus at Vanderbilt University and former Chief Scientist of the Air Force Research Laboratory, and A. R. Ingraffea, Professor at Cornell University. The paper outlines the details of the Digital Twin concept and then discusses the technical challenges that today face the realization of the Digital Twin process.

\section{The Digital Twin Concept}

Consider the following hypothetical capability. In 2025, the United States Air Force (USAF) takes delivery of the first of a new model of aircraft, tail number 25-0001. Along with the physical aircraft, the USAF also receives an asbuilt digital model of this particular aircraft, designated 25$0001 \mathrm{D} / \mathrm{I}$. $25-0001 \mathrm{D} / \mathrm{I}$ is a 1000 billion degree-of-freedom (DOF) hierarchical, computational structures model of 250001. This "Digital Twin" is ultrarealistic in geometric detail, including manufacturing anomalies, and in material detail, including the statistical microstructure level, specific to this aircraft tail number. 25-0001D/I accepts probabilistic input of loads, environmental, and usage factors, and it also tightly couples to an outer-mold-line, as-built, computational fluid dynamics (CFD) model of 25-0001.
25-0001D/I can be virtually flown through a 1 hour flight in 1 hour on an exaflop-scale high-performance computer. During each such virtual flight, 25-0001D/I accumulates usage damage according to the best-physics-based, probabilistic simulations, and outputs about 1 petabyte of material and structural performance and damage data. 25-0001D/I is "flown" for 1000 hours during the design, development, and initial testing of 25-0001. During this accelerated, preliminary "testing", a number of unexpected failure modes are uncovered that would lead to loss of primary structural elements, with 2 incidents projected to result in the loss of the aircraft. Appropriate repairs, redesigns, and retrofits are planned and implemented on 25-0001 before its first flight to preclude such events from actually occurring.

It is recognized, however, that design-point usage is always trumped by actual usage, involving unplanned mission types and payloads. Therefore, a second digital instantiation, $25-0001 \mathrm{D} / \mathrm{A}$, is linked to the structural sensing system deployed on 25-0001. This sensor system records, at high frequency, actual, six-DOF accelerations, as well as surface temperature/pressure readings during each actual flight of 25-0001. Each hour of real flight produces about 1 petabyte of such real data. This data are input into the 25-0001D/A structural model, and this model itself becomes a virtual sensor, interpolating sparse acquired data over the entire airframe. Using Bayesian statistical techniques, $25-0001 \mathrm{D} / \mathrm{I}$ is periodically updated to reflect actual usage of $25-0001$ and recorded in $25-0001 \mathrm{D} / \mathrm{A} .25-0001 \mathrm{D} / \mathrm{I}$ is rerun for forecasting the remaining useful life of 250001, and for updating reliability estimates for all primary structural components. This prognosis leads to time-andbudget-appropriate execution of maintenance, repair, and replacement plans resulting from such updated useful life and reliability estimates. This process is to be executed for all aircraft of this type in the USAF inventory.

The Digital Twin is a reengineering of structural life prediction and management. Is this science fiction? It is certainly an audacious goal that will require significant scientific and technical developments. But even if only a portion of this vision is realized, the improvements in structural life prediction will be substantial. Now consider 
the concept of operation for the Digital Twin process in detail. This will make apparent the technical challenges that need to be overcome to enable the Digital Twin process.

\section{Operation of the Digital Twin}

The operation of the Digital Twin for life prediction would proceed as diagramed in Figure 2. The Digital Twin is represented in the figure as a single entity, but it may consist of several components that are intimately linked, such as a thermal/heat transfer model, a dynamics model, a stress analysis model, and a fatigue model. A mission or series of missions is assigned to a particular aircraft. A reasonable estimate of the flight trajectory and maneuvers that will be flown during the mission is established. The CFD model of that specific aircraft is then "flown" virtually through those flights to estimate the loads and environments the aircraft will experience. As the aircraft is being "flown", aerodynamic pressures on the aircraft are applied to the structural Digital Twin FEM over the time interval of the flight. The CFD and FEM models are closely coupled so that the effect of aeroelastic vibrations and structural deflections on the aerodynamic flow, if any, can be captured, and vice versa. Traditionally, any effect between structural deflections and aerodynamic flows has been discounted, but for a realistic high fidelity model, the possible interactions between physical phenomena should not be ignored from the start.

The structural FEM models the entire range of physics acting on the structure: thermodynamics, global aeroelastic vibrations, and local deformation, both quasistatic and dynamic. The time history of the thermal and stress fields throughout the aircraft is developed for the entire virtual flight. The Digital Twin has full knowledge of how the aircraft has been flown previously and the condition of all structural components in terms of material state and damage at the start of the virtual flight. With these two sets of information, the damage models embedded in the Digital Twin forecast the evolution of material states and the progression of damage during the virtual flight. Damage is not limited to fatigue cracking, but includes creep, fretting and wear, delamination and microcracking in composite materials, corrosion and oxidation, and panel buckling among others. The anticipated probability distribution of remaining useful life for the aircraft is output at the completion of the virtual flight.

Many of the physical phenomena discussed above are nonlinear. As a result, the structural FEM must perform nonlinear analysis. Since the evolution of the material state and the development of damage affect the stiffness of structural components, the coefficient of thermal expansion, and the load at which inelastic deformations begin, among other properties, the material state evolution and damage models must pass information back to the "stiffness matrix" of the FEM so that the thermal and stress fields are accurately determined. In addition, the material state and damage models must communicate with each other so that possible synergistic effects are captured.
Of course, there will be uncertainty about how well the virtual flight reflects what will really happen during the actual flight. In addition, there will always be incomplete information about the properties of the materials used, the quality of the fabrication and assembly methods, and so forth. The Digital Twin will translate these uncertainties in inputs into probabilities of obtaining various structural outcomes. The likelihood of the airframe satisfactorily surviving the demands of the mission can then be factored into whether to send that particular aircraft on that particular mission.

After the physical aircraft flies the actual mission, data about the flight will be downloaded from the aircraft tracking and structural health management (SHm) systems. The aircraft tracking system records sufficient flight parameter data to accurately describe the flight within the CFD Digital Twin. Using the flight parameter history for the actual flight, the Digital Twin is "flown" through the actual mission, and the probability distribution of the actual remaining life is computed.

In addition to having sensors for damage detection at critical locations, the SHm system also senses and records strain histories at select locations during the flight. The strains in the Digital Twin at the selected locations are compared to the strains recorded on the physical aircraft. The damage state at locations in the Digital Twin corresponding to aircraft locations with SHm sensors is compared to the condition detected by the SHm system. Differences between the Digital Twin and the condition of the aircraft are resolved with a formal mathematical process such as Bayesian updating. In this way, the Digital Twin undergoes continuous improvement and becomes more reliable the longer the aircraft is in service.

The use of the Digital Twin is not limited to decisions about a single flight. The usage for a particular aircraft can be projected forward for any desired length of time. The Digital Twin can be flown virtually through all the flights corresponding to the projected usage to forecast the maintenance needs and repair costs for the aircraft during that time. This can be done with the Digital Twin for every aircraft in the fleet to estimate the sustainment needs of the fleet for that period of time. With updating to reflect repairs and part replacements, the Digital Twin can also be used for configuration control of individual aircraft.

The prediction of the remaining useful life of aircraft structure with the Digital Twin requires modeling of the response of the structure to all of the applied loads: quasistatic maneuvering aerodynamic loads, high-frequency sonic and dynamic loads, and thermal fluxes. The time history of the structure's response to these forcing functions must be simulated in order to properly evolve the damage state of the structure with known levels of uncertainty. The damage state at numerous points in the structure must be tracked and updated with each flight. Achieving these requirements is not possible without some significant technical developments in the areas of multiphysics modeling; multiscale damage modeling; integration of the structural and damage models; uncertainty quantification, modeling, and control; manipulation and updating of large 


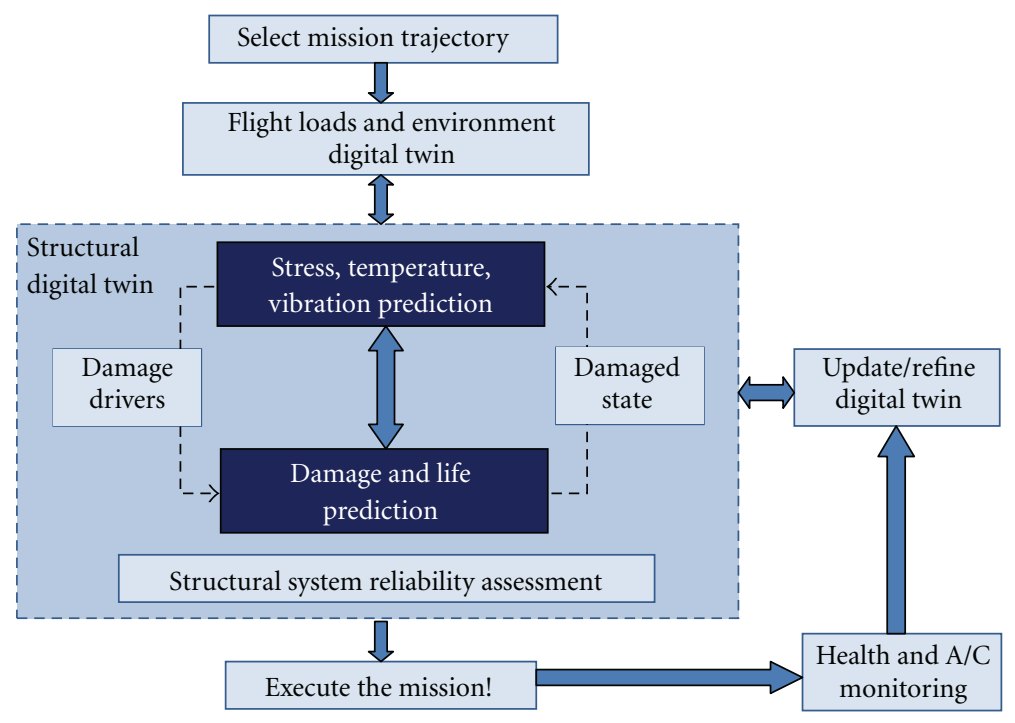

FIgure 2: Functional schematic of the Digital Twin life prediction concept.

databases; high resolution structural analysis. The technical developments required in each of these areas are discussed in the following section.

\section{Needed Capabilities and the State of the Art}

4.1. Multiphysics Modeling. Several commercial structural analysis packages provide the ability to predict thermodynamic response of structures subjected to transient thermal fields, as well as temporally and spatially varying tractions and boundary conditions. These commercial codes perform well for thermal-structural problems that can be addressed using one-way analyses where the thermal stress solution can be computed adequately by performing a thermal analysis followed by a structural analysis using the temperatures derived in the thermal analysis. However, this approach is a simplified model of the actual physics that may not be appropriate for all flight domains.

An example of where two-way coupling may be needed is engine exhaust-washed structure. To prevent "line-of-sight" into the elevated temperature regions of embedded engines, some recent aircraft have the engine exhaust flow over a portion of the airframe in order to give the hot exhaust time to diffuse. This portion of the structure is exposed to a severe thermal-acoustic environment. As the panel deforms out of plane, it is more exposed to the engine exhaust resulting in greater heating. The increased temperature of the skin can increase the out-of-plane deformation further, as well as large changes in the vibration response, resulting in more heating of the panel. The deformation of panel into the exhaust stream can cause separation of the flow from the panel or transition-to-turbulent flow which further affects the heating of the skin. The complex interaction between the physical phenomena cannot be simulated in an one-way coupled analysis.

Two-way coupled methods may be loosely or tightly coupled. Loosely coupled or "partitioned" approaches use two distinct solvers as in the one-way coupling, but now information is passed between solvers at fixed time increments. A tightly coupled, or "monolithic" approach, solves the thermal-structural equations in a single domain via a unified treatment. In general, a tightly coupled method converges more rapidly than the loosely coupled one due to a consistent system tangent stiffness. The disadvantage in the tightly coupled approach is the requirement of additional memory to solve a larger system of equations and accompanying possible ill conditioning of the associated matrices [5].

The most severe challenge for two-way coupling of the physics is the different temporal and geometric scales of the different physics, especially for transient events.

One strategy for solving multiscaled, multiphysics problems is to partition the solution domain along disciplines. Separate techniques can be used for each physical domain at each time interval, while loads, tractions, and other information are exchanged through an interface. Each physical phenomenon has a solver tuned to its particular time and spatial scaling requirements. The key issues with this approach are maintaining coupling and guaranteeing stability, accuracy, and convergence through the interface. The interface needs to apply feedback forces and tractions at the appropriate time and spatial scale without the loss or creation of energy. The interface also has to be constructed so that one physical phenomenon is coupled to another without regard to the peculiarities of the individual solvers.

An example of such an interface method is the common refinement scheme proposed by Jaiman et al. [5]. This method is particularly suited for the mismatched meshes that occur naturally with disparate physical domains. If the interfacial data are not parceled properly with the case of mismatched meshes, accuracy errors can occur. The common refinement scheme integrates over the subintervals to eliminate this error. The scheme allows for the possibility 
of exchanging the solver of either field. For instance, the structural solver could be changed from a full finite element model to a reduced-order model, without influencing the implementation of the acoustic solver. An example of another solution technique is illustrated in Figure 3 for coupled aerothermoelasticity. This particular problem is partitioned into aerothermal and aeroelastic domains, as shown in Figure 3, where the arrows denote the flow of information between the disciplines. This partitioning approach takes into account the differences in required time steps and also allows for different time marching schemes for the different solvers [6]. As shown in Figure 4, the aeroelastic model requires much smaller time steps, $\Delta t_{\mathrm{AE}}$, while the aerothermal model updates the structural temperature distribution at much larger timesteps, $\Delta t_{\mathrm{AT}}$. A disadvantage of the staggered procedure is that accuracy is reduced with increased time.

Another promising method is the combined interface boundary condition (CIBC) procedure [7]. The CIBC procedure appears to have better stability and accuracy characteristics than the staggered scheme, thus addressing two of the main issues of time stepping with partition solvers.

Structures contain regions where locally large gradients occur both spatially and temporally due to the loading. This is also a multiscale issue, as the physical phenomenon causing the gradient occurs at a different scale than is of interest in the structural scale analysis. Conventional FEM practices require increasing the degrees of freedom (DOFs) in regions where spatial resolution is desired and a decrease in the time increment when an increase in the temporal resolution is required. However, as noted in [8], "the tyranny of scales will not be defeated simply by building bigger and faster computers. Instead, we will have to revamp the fundamental ways we conceive of scientific and engineering methodologies, long the mainstays of human progress". Increasing the number of DOFs might lead to convergence with respect to the energy norm $\left(L_{2}\right)$; however, the same is not true concerning local error. In recent work by O'Hara et al. $[9,10]$, a $100 \%$ error in the maximum temperature was obtained for a converged conventional FEM solution for a problem involving a sharp thermal gradient as shown in Figure 5. Structure upon which a shock wave impinges could experience a sharp thermal gradient similar to this. Conventional techniques are unable to recover the imposed boundary conditions in regions of high gradients. This influences the displacements, stresses, and strains resulting in incorrect life and risk estimates. The "tyranny of scales" is more problematic for combined physics problems such as fluid-thermal-structure interactions and where long time records are desired. Recall that the Digital Twin will require simulating a flight of an hour or more in actual time.

Methods being developed to specifically address the multiscale analysis of steep gradients include: the generalized finite element method (GFEM), the space-time method, and reduced-order models (ROMs). Each method offers the capability to introduce necessary physical phenomena at a level of accuracy that cannot be achieved with conventional finite element methods for structural scale analysis. All three computational methods are active research areas for fluid, thermal, and structural disciplines, providing a commonality of approach across disciplines. All three methods promise the advantage of reducing model complexity and computational time.

GFEM uses a priori knowledge of the solution to augment the standard FE shape functions with "enriched" shape functions that capture the physical phenomenon of interest. GFEM uses concepts from the partition of unity [11], where standard FE shape functions are enriched in the master element allowing for the use of existing infrastructure and algorithms from classical finite element methods. GFEM has been applied to fracture mechanics [12], thermal analysis $[3,9]$, and CFD [13] problems. However, three research issues remain in order for GFEM to address real aerospace situations. The thermal example in Figure 5 is a simple problem with a static gradient. In general, the gradients in the structure are changing in magnitude and location during a flight. Therefore, different elements in the FEM will need enrichment at different times during a flight. Research is also needed on the type of enrichment functions that are best able to capture the gradients for computational efficiency. Finally, given an accurate representation of a thermal gradient, how should the structural shape functions be enriched to accurately calculate strains and stresses in the region if the thermal gradient is unknown?

Space-time methods assume that the time dimension can be treated in the element domain using interpolation functions as is customary in the spatial domain; that is, approximations are established in both space and time. The first space-time application can be traced to Oden in 1969, where it was applied to wave propagation in a bar [8]. More recently, space-time methods have been applied in formulating a fully coupled framework to address acousticstructure interaction as well as many other applications requiring self-adaptive solution strategies to track transient waves propagating spatially and temporally, such as those occurring in fluid-structure interactions [14]. The algorithm is unconditionally stable and is not limited by the critical time step. Finally, the space-time method provides a framework for improving the solution accuracy through enrichment. This is particularly promising for historydependent life prediction.

While space-time methods show promise in addressing transient dynamic phenomena, there are disadvantages associated with the method. Besides adding complexity in the element formulation, the size of the global stiffness matrix is $n^{2}$, where $n$ is the number of interpolation points in time. For example, a linear element in time would require four times the global stiffness size of a semidiscrete time formulation and nine times the size for a quadratic formulation. This greatly increases the computational costs associated with the space-time procedure and may no longer provide a benefit for many typical problems solved with finite elements.

ROMs reduce the computational burden of direct time integration of an FEM with random dynamic loading prescribed, while retaining the necessary accuracy. Direct time 




FIGURE 3: Aerothermoelastic coupling approach for the hypersonic environment [1].

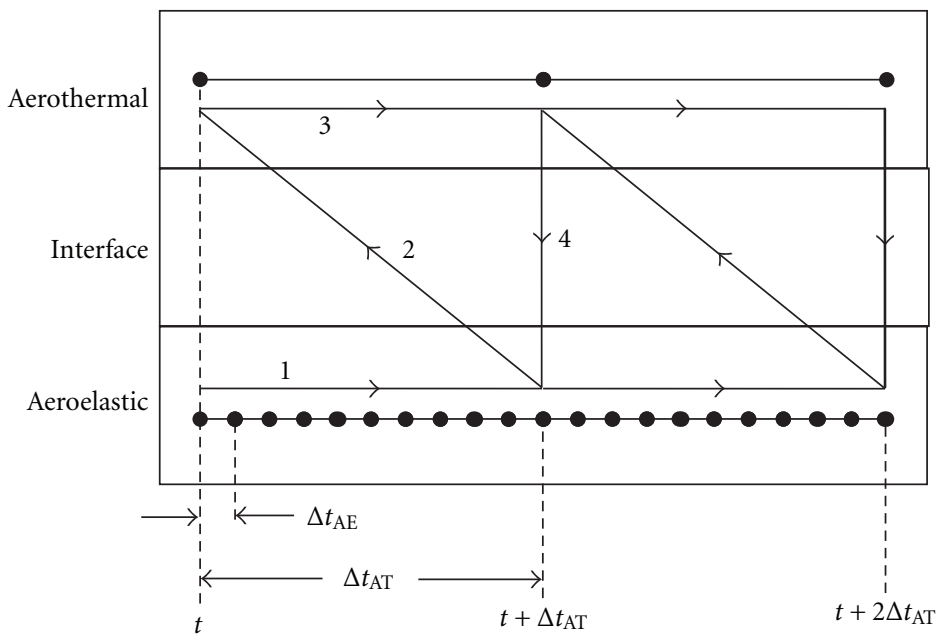

Figure 4: Aerothermoelastic partitioning approach [2].

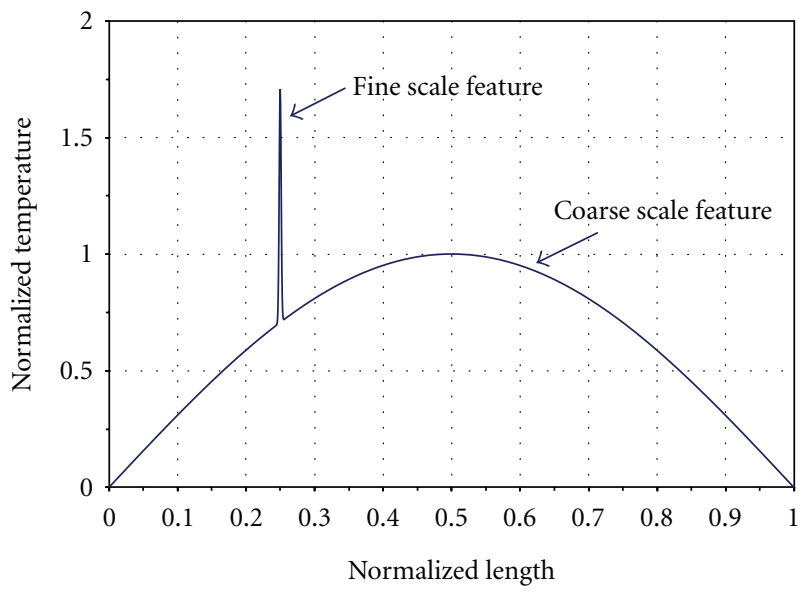

FIgURE 5: Coarse scale feature plus a fine scale feature with a large local gradient [3]. 
integration of a standard, large-order FEM for a missionscale length of time can be cost and time prohibitive.

The use of nonlinear structural ROMs, particularly for acoustic fatigue type response, is well documented in the literature [15-18]. Further, adaptation of nonlinear structural ROMs to coupled thermal and deformation problems through the use of "cold modes" has been proposed [13, $14,19]$. These schemes use the linear mode shapes of the structure in the so-called unstressed or cold state to span the response at different temperature states. The linear portions of the structural ROM become functions of temperature while the nonlinear terms remain constant. Falkiewicz and Cesnik [20] describe a thermal ROM based on proper orthogonal decomposition and the method of snap shots that was used to update the temperature distribution in the structural ROM via the thermal flux. Both the structural and thermal ROMs were linear. Coupled ROMs have also been used to create a unified aeroelastic and flight dynamic formulation [21].

A ROM will likely be needed to provide the aerodynamic loads over an entire flight. CFD is normally used to simulate an aircraft in cruise conditions, not as it accelerates and maneuvers [22]. While some non-steady-state flight conditions such as recovery of an F-18 over a carrier deck [23] and a cargo airdrop from a C-130 [24] have been simulated with overset grid methods [25], longitudinal accelerations of an aircraft are still a challenge. In CFD, velocity changes to the fluid flow are introduced at one side of the meshed region and propagate across the region with time. However, when an aircraft changes airspeed, the air surrounding the aircraft changes speed relative to the aircraft at once.

4.2. Multiscale Damage Modeling. The Digital Twin will mimic the behavior of its actual aircraft as much as possible. Assuming damage of specific sizes at all the critical locations, as is done in damage tolerance requirements, is not in keeping with this intent. Damage tolerance checks for slow crack growth or fail safety will still be performed to ensure safety. However, only known damage will be placed in the Digital Twin for life management purposes. If damage is not known to exist at a location, a distribution of damage forming features, such as those in Figure 6 for fatigue cracks, will be assumed at that location.

The distribution and type of damage forming features will be dependent upon the material and fabrication process. Physics-based models for how damage forms from these features will need to be integrated into the structural FEM. Figure 6 is a schema for the stochastic information flow back and forth between the material and structural scales of a nondeterministic life prediction for fatigue cracking.

Figure 6 shows the conceptual data flow through the use of actual component- and material-scale imagery and actual and surmised associated statistics [26, 27]. An assessment of reliability against a fracture limit state starts with a proposed component-scale structural design, Figure 6(a). A stress analysis is performed, and fatigue "hot-spots" located in the component. This assessment is stochastic, because it has to consider uncertainties in boundary conditions, geometry, usage, and environment. Statistically representative microstructure-scale volume elements, such as that depicted in Figure 7, are then created at each hot spot.

As shown in images in Figures 6(b) through 6(f), the physics and mechanics of the incubation, nucleation, and microstructurally small fatigue crack (MSFC) processes and events must be known, incorporated in predictive software and used to produce the relevant statistics for each stage of crack growth. In this particular example, the physics and mechanics of incubation and nucleation in 7075-T651 are associated with $\mathrm{Al}_{7} \mathrm{Cu}_{2} \mathrm{Fe}$ constituent particles, and a set of 4 such particles are hypothetically tracked through the cracking events and processes. At any given stage, those particles outlined in red are participating in that stage; particles outlined in black are inactive. Incubation is the period before a crack in a particle penetrates into the aluminum matrix. Nucleation is when the crack in a particle first starts in the matrix as indicated by the arrows in the upper photographs in Figure 6(e). MSFC propagation occurs as the crack grows away from the particle and navigates across a number of grains to a size at which traditional component scale fatigue models become applicable.

By the image in Figure 6(f), a distribution of MSFC growth rates has been produced. MSFC propagation is governed by (as yet not well known) rules for crack growth rate, direction, shape, grain boundary interaction, and coalescence. Screening determines which of the nucleated cracks grow at a rate below a threshold and would deem these microcracks inactive, while the other, active, microcracks would be further considered for long crack growth simulation. Traditional, structural-scale fatigue crack growth methods are then applied to predict the remaining number of cycles to failure. Using the statistics accrued through the course of the modeling approach, a distribution of life, or reliability, is finally determined, Figure $6(\mathrm{~g})$.

Similar schemata need to be developed for other damage processes and for other materials such as composites or hybrids. The multiscale physics models for thermomechanical fatigue, creep, fretting and wear, corrosion and oxidation, and delaminations and microcracking of composite materials need to be developed for aircraft structural materials. The possibility of synergistic interactions between damage mechanisms at all scales must also be explored. The effort that went into developing the level of understanding of fatigue crack formation in 7075-T651 depicted in Figure 6 was phenomenal by any standard. Developing these models for all of these other damage mechanisms and for every aircraft structural material in any reasonable period of time will require an investment on the order of the DARPA Accelerated Insertion of Materials program [28].

\subsection{Integration of Structural FEM and Damage Models.} It is important to understand that each stage of crack growth, or other damage process, is driven by stochastic driving forces computed at the component scale and that the component-scale model itself will periodically have to be updated to account for material-scale damage that reduces local stiffness resulting in redistribution of local 


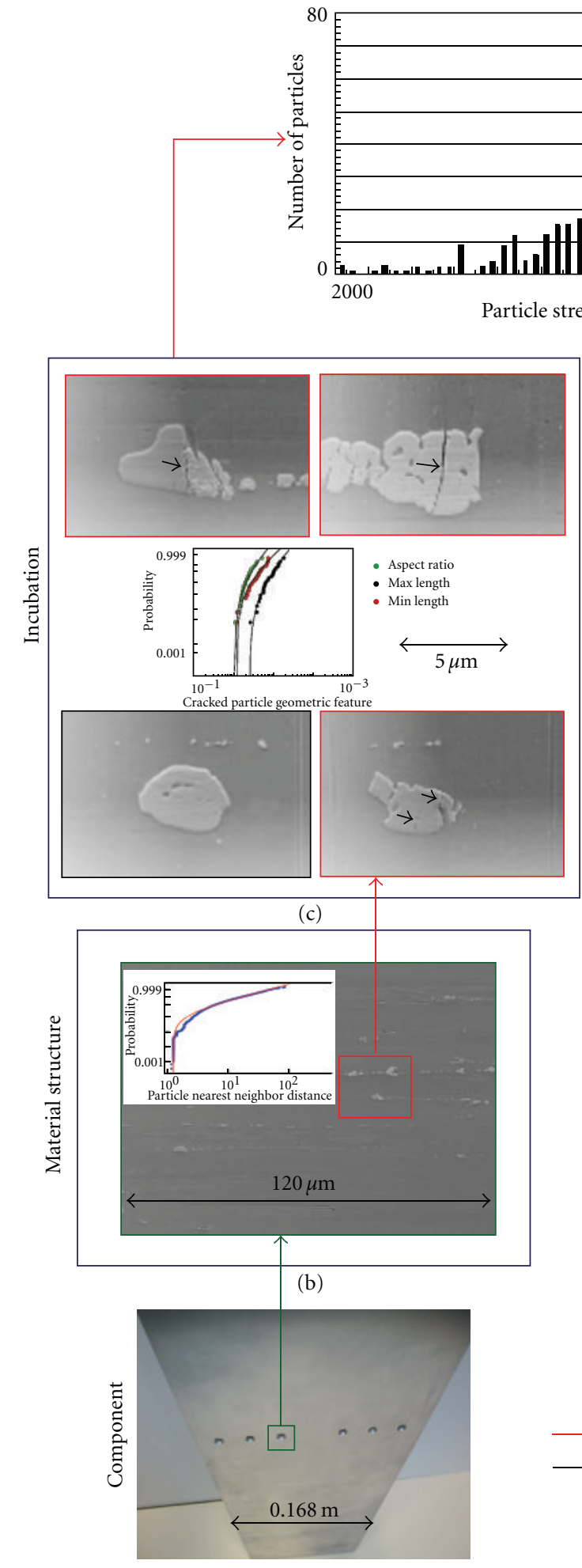

(a)

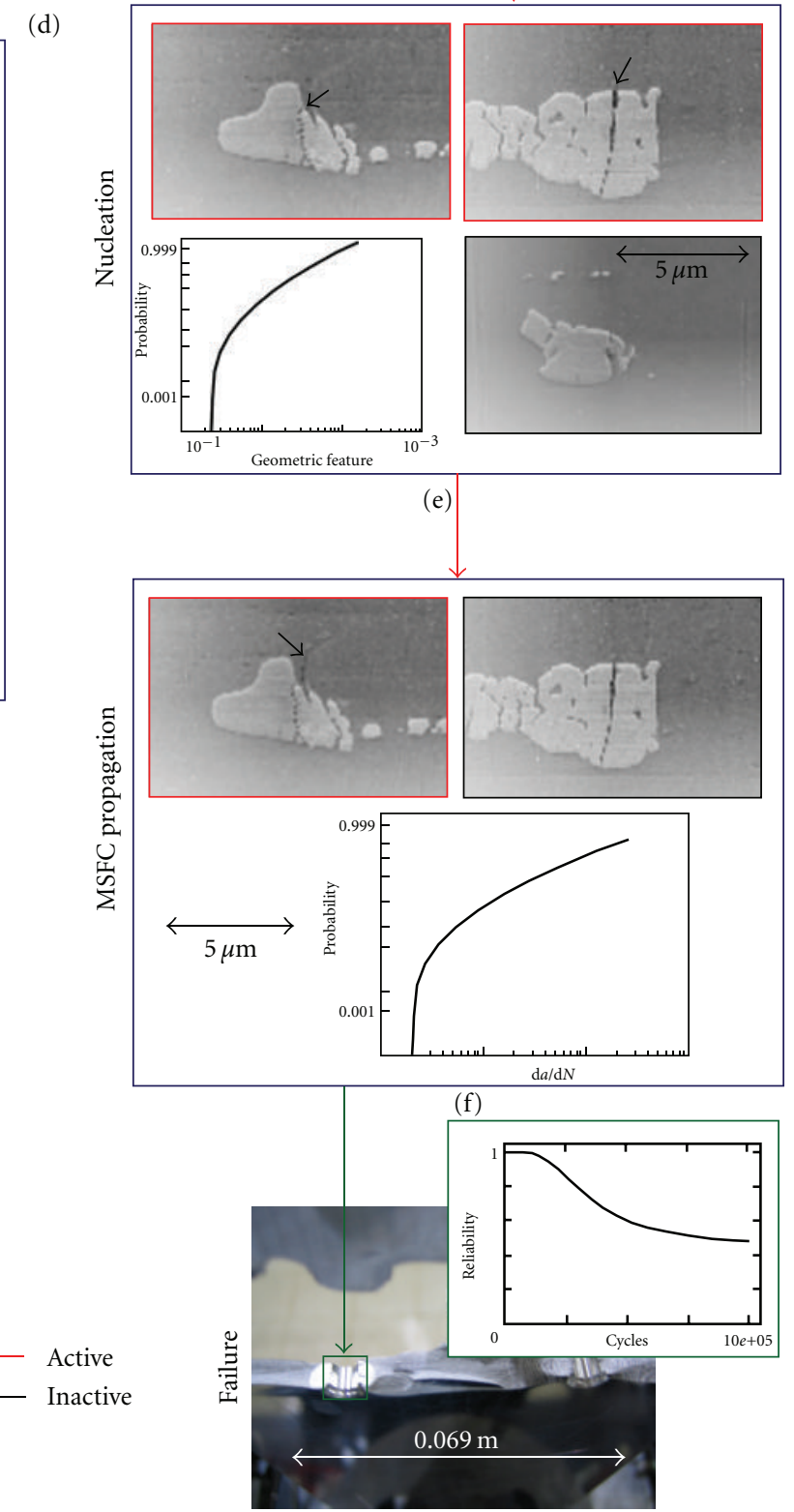

(g)

FIGURE 6: Schematic of stochastic information flow in a coupled material-scale, structural-scale simulation environment for a traditional metallic component. The predicted probability and statistics of microstructural information and behavior reveal a limit state in, and inform the reliability of, a structural component. 




FIGURE 7: Finite element model of polycrystal of 7075-T651 for microstructural studies of fatigue mechanisms from particle inclusions: 340 PE's, 33 million DOF.

stress and thermal fields. The need for a stochastic, multiscale simulation capability that integrates material performance with structural response becomes obvious.

Employing traditional cycle-based fatigue models in the Digital Twin, besides adding significant memory and storage requirements to an already large model, will contribute to uncertainty by allowing updates to the damage state of the structural model only after the completion of a stress cycle. When the damage state at a location is finally updated, there is a step function change in the local stiffness and resulting redistribution of stress. However, because damage has been developing in the physical aircraft all during the stress cycle, the stiffness change has been continuous and gradual, as has the redistribution of stress. Thus the damage from the stress cycle may not be as much as the cycle-based model would predict because the stress redistribution had reduced the amplitude of the stress cycle.

Cycle-based models are a historical remnant of the time when experimental and numerical modeling capabilities were more constrained than today. With the ability to record video images at scanning electron microscope scales and process those images to measure small displacements, fatigue crack growth increments can be determined more frequently than every few hundred cycles. Improvements in the hardware and software for numerical modeling make it easier to solve differential equations for which the time discretization is smaller than a stress cycle. There is no reason why models relating the time rate of change of damage to the time rate of change of the stress cannot be developed today. Small time scale fatigue crack growth models have been proposed that compute the increment of crack growth at any time instant [29-31]. These models were developed from the standard cyclic fracture mechanics relationship for crack growth. The models were able to represent cyclic crack growth data under constant and variable amplitude loading without having to count cycles or track crack closure levels. Such models will facilitate the integration of damage models into structural FEMs.

The challenge of establishing a two-way coupling between a structural FEM and a damage model still remains even if the damage model is time based. One approach to integrating a material damage model into a structural FEM is continuum damage mechanics. Continuum damage mechanics approaches the local failure of materials by introducing internal state variables to quantify the local degradation at a material point. The effect of this degradation is included in the stiffness equations for the material in the FEM. Successful application of continuum damage mechanics is dependent upon the formulation of the evolution equation for the state variables. Void growth models are the most common damage evolution models [32], but continuum damage mechanics has been applied to subcritical crack growth under monotonic loading [33]. While the evolution equations can be formulated using a phenomenological procedure, a multiscale homogenization procedure using the physics-based damage models discussed above would be preferred.

Partition of unity methods, such as GFEM and extended FEM (XFEM), are also promising approaches for integrating damage into the structural FEM. For instance, these methods allow the representation of discontinuities and singularities via geometric descriptions of the crack surfaces, which are independent of the volume mesh, coupled with suitable enrichment functions [34]. In other words, a single finite element mesh suffices for modeling as well as capturing the evolution of material boundaries and cracks because the finite element mesh does not need to conform to internal boundaries.

Both approaches have been successful at integrating damage into a structural FEM when there is a single dominant damage driver, typically stress or strain. Simulation of damage development in more complex situations involving varying temperature, environment, and stresses is currently limited by the development of suitable damage state evolution equations.

4.4. Uncertainty Quantification, Modeling, and Control. The purpose of quantifying uncertainty in a model is to exercise some control over the magnitude of the uncertainty [35, 36]. The magnitude of the uncertainty can be controlled by the choice of model fidelity and scale. It is not given that the finest analysis scale and highest fidelity models decrease uncertainty in the simulation results sufficiently to justify their cost. There may be input parameters, such as the applied loading, whose uncertainty overrides all of the other uncertainties in the model. Higher fidelity models and grain-scale analyses will not decrease the uncertainty associated with the applied loading. Computational cost increases as finer scales are analyzed and higher fidelity models are used. The choice of what fidelity and scale to use in a simulation should be based upon the acceptable level of uncertainty and the computational cost to achieve it. The 
ideal situation is to know the impact of scale and fidelity choices on uncertainty prior to performing any simulations. Determining, after the effort, that a simulation did not provide an acceptable level of uncertainty is of less benefit. The cost of one simulation has already been incurred, and now another simulation of finer scale and higher fidelity will need to be performed with no guarantee that it will provide an acceptable level of uncertainty either.Sampling methods, such as Monte Carlo simulations and variations thereof, are commonly used to determine the uncertainty/variability in results. However, performing Monte Carlo simulations with different realizations of a model of an entire aircraft over a complete flight brings significant computational cost. Modeling and simulation of an entire airframe is computationally intensive even with today's highly idealized elastic models at selected points in the flight spectrum. A detailed, nonlinear simulation of an entire flight for a complete airframe amplifies the computational time by orders of magnitude. Monte Carlo simulations would require analyses of tens, if not hundreds, of realizations "flying" the same mission in order to obtain just the first and second moments of the distribution for each output variable. And, while sampling methods are readily parallelized, more sophisticated probabilistic methods such as stochastic finite element methods (SFEMs) might be better than sampling methods.

SFEM comprises three basic steps: discretization of the stochastic fields representing the uncertain input parameters, formulation of the stochastic matrix at the element and then at the global level, and, finally, the response variability calculation [37]. There are two main variants of SFEM: the perturbation approach based on a Taylor series expansion of the response vector and the spectral stochastic finite element method, where each response quantity is represented by a series of random Hermite polynomials. Each of these variants has issues in terms of computation effort that make application to large-scale non-linear systems, such as an aircraft, currently prohibitive.

There are other developments in SFEM that hold promise. The first is stochastic reduced basis methods (SRBMs) where the response process is represented using basis vectors that span the preconditioned, stochastic, Krylov subspace [38]. SRBMs are computationally efficient as compared to polynomial chaos expansions (PCEs) at a comparable level of accuracy, and so are better suited for solving large-scale problems. The computational costs of simulations using PCEs are such that solutions have been limited to uncertain systems with a small number of degrees of freedom. The SRBM formulation is limited to the analysis of random linear systems at this time, as is the PCE formulation. Furthermore, the basis vectors are problem dependent which limit the ability to develop a general approach.

The second development is nonintrusive SFEM approaches [39]. These approaches take advantage of powerful existing deterministic FE codes by building a surrogate response surface model using PCEs. The ability to use any third party FE code speeds the development and transition of SFEM by removing the need to develop an entire SFEM system from scratch. The investments in deterministic FE codes can be leveraged.

Other developments of interest include multiscale SFEM [40] and an extended SFEM (X-SFEM) analogous to XFEM for deterministic FEM [41]. Multiscale SFEM seeks to propagate uncertainty information in fine scale quantities, such as microstructure, to coarse scale quantities, such as stiffness and strength that are functions of the fine scale quantities. X-SFEM provides for the propagation of geometric uncertainties in the solution of partial differential equations, that is, PDEs defined on random domains.

4.5. Manipulation of Large, Shared Databases. A model of an entire airframe is, by itself, an enormous database that is difficult to input, maintain the integrity of, and manipulate. The basic geometry and assembly of components for the airframe can be established with a CAD system. Discretization of the individual components can be challenging, especially for large, detailed structural components. The integrity of the geometry and the discretization of this large complex model must be established and maintained over the life of the model. The discretization must be adaptable in order to adequately model the insertion of unexpected damage and subsequent repairs that occur during the service life of an aircraft. These tasks will likely need to be automated as current manual methods are not up to the Digital Twin challenge.

The information generated by performing flight-byflight simulations over the entire design or service, life of an aircraft will be voluminous. In fact, manipulation of just the information from the simulation of a single flight for an entire air vehicle stretches current capabilities. For the Digital Twin, the results from the simulation of every flight during the life of the aircraft must be kept available. In order to use this information for making decisions about the continuing airworthiness of the vehicle, all of the information contained in the simulation results must be accessible. Rapid, focused interrogation of the database to support specific decisions must be possible. Some interrogations will need to be automated. For instance, it is not practical to manually search through an entire aircraft, physically or virtually, to locate damage hot spots. To visualize $1 \%$ of 1 petabyte of data envisioned as the output of a virtual flight will take 35 workdays at the current rate of $10 \mathrm{MB} / \mathrm{s}$ [4]. The Digital Twin will need to automatically identify locations with prescribed levels of damage and present this information in a userfriendly way.

4.6. High-Resolution Structural Analysis Capability. Simulation-based design and certification will require very high performance computing, performance far beyond what is commonly used for aircraft structural analyses today. As envisioned, the Digital Twin of a complete airframe will have on the order of $10^{12}$ degrees of freedom. If multiscale models of the microstructure, such as in Figure 7, are required at some locations, these models will have on the order of $10^{7}$ degrees of freedom at each location [26]. Despite its large size, the Digital Twin must execute with sufficient speed so that the modeling and simulation can keep up with the actual 


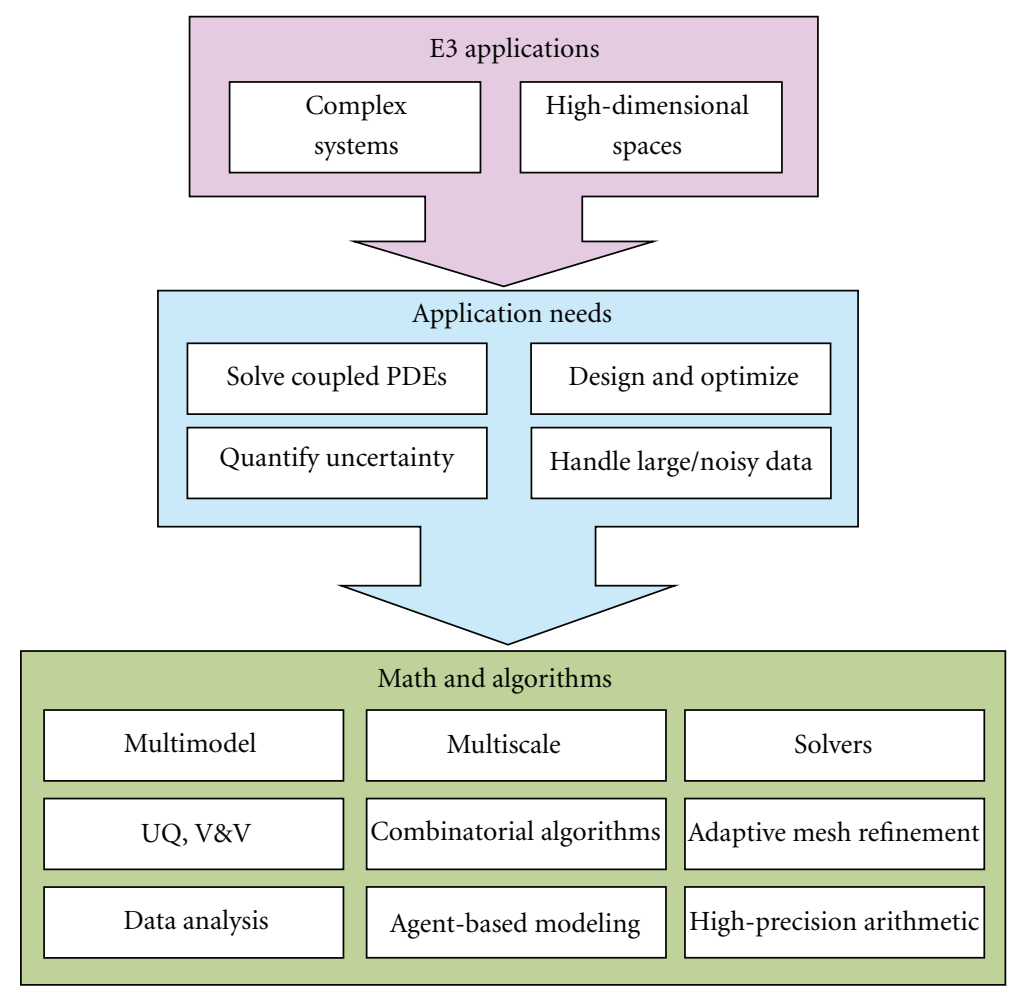

FIGURE 8: Common applications characteristics and math and algorithms needs for problems like high-resolution structural simulation [4].

usage of the aircraft, that is, a 1-hour flight must be simulated in 1-hour of clock time or less. If simulations are unable to stay ahead of the actual aircraft, the power of the Digital Twin for life prediction and decision making is lost. Clearly, very high performance computing will be needed to meet the vision of the Digital Twin.

But high-performance computing (HPC) is a relative term. In the next several years, petaflop-per-second power will become available. Within a decade, it is expected that exaflop-per-second computers will become available:

\section{"extrapolation of current hardware trends suggests that exascale systems could be available in the marketplace by approximately 2022 via a "busi- ness as usual" scenario. With the appropriate level of investments, it may be possible to accelerate the availability by up to five years, to approximately $2017 "$ [4].}

The teraflop-per-second scale computing of today is effectively infinite computing power by the standard of many engineers. The problem is not the availability of highperformance computing hardware, but rather the usability of it through the appropriate codes and other software tools. A recent U.S. Department of Defense survey [42] found that the average age of commercially available finite element software is about 20 years and that the typical maximum number of processors such tools could effectively access was about 300! The gap between hardware capability and software performance is recognized by the HPC community [4]: "advanced and improved simulations ... demand significant advances in mathematical methods, scalable algorithms, and their implementations. The required advances are driven by the increased complexity of the problems, involving multiple and coupled physics models, high dimensionality described by large numbers of equations (PDEs, ODEs, DAE, geometric descriptions and boundary conditions, optimization, etc.), and by the huge time and spatial scales..."

The Digital Twin is typical of what is called an "E3 Application", a complex system in high-dimensional spaces. The HPC community has identified certain application characteristics and math and algorithms needs (Figure 8) which have already been discussed for the Digital Twin. Within the context of the Digital Twin, there is a need to solve coupled PDEs, quantify uncertainty, design and optimize the structure, while handling large and noisy data.

The HPC community has accurately described the computational problems of the Digital Twin. Solving very large systems of PDE's with uncertainty everywhere is at the core of the Digital Twin concept.

DoD has taken steps to address this need through their Computational Research and Engineering Acquisition Tools and Environments (CREATE) Program. Specifically the air vehicle portion has developed a fixed wing virtual aircraft simulation tool called Kestrel [43]. Kestrel integrates multiple single executable modules. The most significant for the purposes of the Digital Twin are a CFD solver and a linear modal representation of the aircraft along with 
fluid-structure interfacing operations. Aerodynamic loads are applied directly to the structure. However, the stresses in the structural components still need to be determined to enable life prediction.

\section{Developing the Digital Twin}

There are many challenges that must be overcome in developing the Digital Twin. It is difficult to put together a comprehensive Digital Twin development plan that covers a decade or more of activities. However, the initial, pathfinding work that has been done and that is planned for the near future will be discussed.

The Air Vehicles Directorate at the U.S. Air Force Research Laboratory has been investigating a ROM for obtaining aerodynamic loads on the aircraft or internal stresses, from pilot inputs either in the actual aircraft or in a flight simulator. This activity developed out of work to streamline the clearance for external stores on an aircraft [21, 44]. The integration of this stick-to-stress ROM into structural life prediction will be investigated as part of a program to demonstrate the potential of higher fidelity stress history, structural reliability analysis, and structural health monitoring for improving the management of airframes. Two full scale fatigue tests of aircraft assemblies will serve as surrogates for actual flying aircraft in this program. The result of this program will be an initial, low fidelity "Digital Twin".

Additional spirals of development will increase the fidelity of this Digital Twin by incorporating new technologies as they mature. One of the technologies that may be ready in time for the second development spiral includes physics-based models for damage development and progression from NASA's Damage Sciences [45] and the Air Force Office of Scientific Research's Structural Mechanics programs. Another may be the coupling of different physics models, that is, thermal, dynamic, and stress. This topic is being actively worked by the Air Vehicles' Structural Sciences Center $[1,2]$.

A third technology is the digital thread manufacturing technology [46] for the F-35. The digital thread makes it easier to see how the information necessary to construct tail number-specific structural models can be collected. In the digital thread, the same 3D solid models from engineering design are used in manufacturing for numerically controlled programming, coordinate measurement machine inspections. Laser measurements are used with the digital thread to virtually mate parts in order to identify potential fit-up problems prior to actually mating the parts. The digital thread, in addition to the F-35 production rates, has enabled Lockheed to use automated hole drilling in many places. Therefore, to within the accuracy of the production measurement systems, the dimensions of many of the detail parts and the location of many of the fastener holes were known at one time during production. It becomes a matter of supplying that information as initial conditions to the Digital Twin for an aircraft as it enters service.

\section{Advantages of a Digital Twin}

In the current life prediction process for aircraft, each type of physics has its own separate model. There is the computational fluid dynamics (CFD) model, the structural dynamics model (SDM), the thermodynamic model, the stress analysis model (SAM), and the fatigue cracking model (FCM). Computational capabilities have restricted what physics and damage models are considered during the life prediction process. Information is passed between the physics models by writing the results from one model to a file, translating that output file into an input file for the other model, and finally reading the input file into the second model. This process makes it difficult to develop a synchronized stress-temperature-chemical (STC) loading spectrum. Furthermore, the effect of damage development on the stress or temperature history is not considered. The approach has been to assume some appropriately severe conditions during the design and subsequent usage tracking for an aircraft. Such an approach is usually conservative, but leads to an air vehicle that is heavier than it may need to be and inspections more frequently than may be needed.

With the Digital Twin, the SDM, the SAM, the FCM, and possibly other material state evolution models would be integrated into a single unified structural model that is tightly coupled to a CFD Digital Twin. The physics involved would be seamlessly linked, the way that physics is linked in the physical structure. The joint STC loading history for the aircraft will directly result from the simulation of the flight. This joint spectrum can be found for any location in the structure and will not rely on an idealized transfer function. As damage develops within the structure, the local STC spectrum will naturally adjust for the presence of damage. It will not be necessary to assume the repetition of a statistically representative spectrum over the lifetime of the vehicle either; the STC spectrum can evolve as the usage of the vehicle and the age of the structure dictate.

Damage findings, repairs, replacements, and structural modifications, if they are recorded at all, are presently maintained in a database separate from the structural analysis models. It is not clear that this database is consulted when updating the remaining useful life of an aircraft. Such information is certainly not stored in a format that facilitates its use in a structural analysis model. The Digital Twin would provide a visual database that is directly related to both the structural model and the physical aircraft. Therefore, in addition to providing a structural life prediction tool, the Digital Twin also facilitates configuration control for an individual aircraft.

The Digital Twin will enable better management of an aircraft throughout its service life. Engineers will have more information about the condition of the aircraft and have it sooner. This will allow better maintenance decisions to be made in a timely manner.

\section{Disclosure}

This paper was cleared for public release-Distribution Aon 29 July 2011 as case 88ABW-2011-4181. 


\section{References}

[1] B. A. Miller, J. J. McNamara, A. J. Culler, and S. M. Spottswood, "The impact of flow induced loads on snapthrough behavior of acoustically excited, thermally buckled panels," in Proceedings of the 51st AIAA/ASME/ASCE/ AHS/ASC Structures, Structural Dynamics and Materials Conference, Orlando, Fla, USA, April 2010, AIAA-2010-2540.

[2] A. J. Culler, A. R. Crowell, and J. J. McNamara, "Studies on fluid-structural coupling for aerothermoelasticity in hypersonic flow," in Proceedings of the 50th AIAA/ASME/ASCE/ AHS/ASC Structures, Structural Dynamics and Materials Conference, Palm Springs, Calif, USA, May 2009, AIAA-2009-2364.

[3] R. Merle and J. Dolbow, "Solving thermal and phase change problems with the extended finite element method," Computational Mechanics, vol. 28, no. 5, pp. 339-350, 2002.

[4] H. Simon, T. Zacharia, and R. Stevens, Modeling and Simulation at the Exascale for Energy and the Environment, Office of Science, U.S. Department of Energy, 2007, http:// www.sc.doe.gov/ascr/ProgramDocuments/ProgDocs.html.

[5] R. K. Jaiman, X. Jiao, P. H. Geubelle, and E. Loth, "Assessment of conservative load transfer for fluid-solid interface with non-matching meshes," International Journal for Numerical Methods in Engineering, vol. 64, no. 15, pp. 2014-2038, 2005.

[6] C. A. Felippa, K. C. Park, and C. Farhat, "Partitioned analysis of coupled mechanical systems," Computer Methods in Applied Mechanics and Engineering, vol. 190, no. 24-25, pp. 3247-3270, 2001.

[7] B. Roe, R. Jaiman, A. Haselbacher, and P. H. Geubelle, "Combined interface boundary condition method for coupled thermal simulations," International Journal for Numerical Methods in Fluids, vol. 57, no. 3, pp. 329-354, 2008.

[8] J. T. Oden, "A general theory of finite elements II. Applications," International Journal for Numerical Methods in Engineering, vol. 1, no. 3, pp. 247-259, 1969.

[9] P. O'Hara, C. A. Duarte, and T. Eason, "Transient analysis of sharp thermal gradients using coarse finite element meshes," Computer Methods in Applied Mechanics and Engineering, vol. 200, no. 5-8, pp. 812-829, 2011.

[10] P. O’Hara, C. A. Duarte, and T. Eason, “Generalized finite element analysis of three-dimensional heat transfer problems exhibiting sharp thermal gradients," Computer Methods in Applied Mechanics and Engineering, vol. 198, no. 21-26, pp. 1857-1871, 2009.

[11] C. A. Duarte, I. Babuška, and J. T. Oden, "Generalized finite element methods for three-dimensional structural mechanics problems," Computers and Structures, vol. 77, no. 2, pp. 215232, 2000 .

[12] C. A. Duarte and D. J. Kim, "Analysis and applications of a generalized finite element method with global-local enrichment functions," Computer Methods in Applied Mechanics and Engineering, vol. 197, no. 6-8, pp. 487-504, 2008.

[13] L. T. Zhang, G. J. Wagner, and W. K. Liu, "A parallelized meshfree method with boundary enrichment for large-scale CFD," Journal of Computational Physics, vol. 176, no. 2, pp. 483-506, 2002.

[14] L. L. Thompson and P. M. Pinsky, "A space-time finite element method for structural acoustics in infinite domains part 1: formulation, stability and convergence," Computer Methods in Applied Mechanics and Engineering, vol. 132, no. 3-4, pp. 195227, 1996.

[15] A. Przekop and S. A. Rizzi, "Nonlinear reduced order random response analysis of structures with shallow curvature," AIAA
Journal, vol. 44, no. 8, pp. 1767-1778, 2006.

[16] A. Przekop and S. A. Rizzi, "Dynamic snap-through of thinwalled structures by a reduced order method," in Proceeding of the 47th AIAA/ASME/ASCE/AHS/ASC Structures, Structural Dynamics and Materials Conference, pp. 1853-1863, Newport, RI, USA, May 2006, AIAA-2006-1745.

[17] J. J. Hollkamp, R. W. Gordon, and S. M. Spottswood, "Nonlinear modal models for sonic fatigue response prediction: a comparison of methods," Journal of Sound and Vibration, vol. 284, no. 3-5, pp. 1145-1163, 2005.

[18] B. Yang, M. P. Mignolet, and S. M. Spottswood, "Modeling of damage accumulation for Duffing-type systems under severe random excitations," Probabilistic Engineering Mechanics, vol. 19, no. 1, pp. 185-194, 2004.

[19] X. Q. Wang, M. P. Mignolet, T. G. Eason, and S. M. Spottswood, "Nonlinear reduced order modeling of curved beams: a comparison of methods," in Proceedings of the 50th AIAA/ASME/ASCE/AHS/ASC Structures, Structural Dynamics and Materials Conference, Palm Springs, Calif, USA, May 2009, AIAA-2009-2433.

[20] N. J. Falkiewicz and C. E. S. Cesnik, "A reduced-order modeling framework for integrated thermo-elastic analysis of hypersonic vehicles," in Proceedings of the 50th AIAA/ASME/ASCE/AHS/ASC Structures, Structural Dynamics and Materials Conference, Palm Springs, Calif, USA, May 2009, AIAA-2009-2308.

[21] D. H. Baldelli, P. C. Chen, and J. Panza, "Unified aeroelastic and flight dynamic formulation via rational function approximations," Journal of Aircraft, vol. 43, no. 3, pp. 763-772, 2006.

[22] D. Garretson, H. Mair, C. Martin, K. Sullivan, and J. Telchman, "Review of CFD capabilities," DTIC Accension Number ADA 537587, Institute for Defense Analysis, 2005.

[23] J. D. Shipman, S. Arunajatesan, P. A. Cavallo, and N. Sinha, "Dynamic CFD simulation of aircraft recovery to an aircraft carrier," in Proceedings of the 26th AIAA Applied Aerodynamics Conference, Honolulu, Hawaii, USA, August 2008, AIAA2008-6227.

[24] R. W. Noack, "SUGGAR: a general capability for moving body overset grid assembly," in Proceedings of the 17th AIAA Computational Fluid Dynamics Conference, Ontario, Canada, 2005, AIAA-2005-5117.

[25] K. Nakahashi, Y. Ito, and F. Togashi, "Some challenges of realistic flow simulations by unstructured grid CFD," International Journal for Numerical Methods in Fluids, vol. 43, no. 6-7, pp. 769-783, 2003.

[26] J. E. Bozek, J. D. Hochhalter, M. G. Veilleux et al., "A geometric approach to modeling microstructurally small fatigue crack formation: I. Probabilistic simulation of constituent particle cracking in AA 7075-T651," Modelling and Simulation in Materials Science and Engineering, vol. 16, no. 6, Article ID 065007, 2008.

[27] J. M. Emery, J. D. Hochhalter, P. A. Wawrzynek, G. Heber, and A. R. Ingraffea, "DDSim: a hierarchical, probabilistic, multiscale damage and durability simulation system-part I: methodology and Level I," Engineering Fracture Mechanics, vol. 76, no. 10, pp. 1500-1530, 2009.

[28] Integrated Computational Materials Engineering-A Transformational Discipline for Improved Competitiveness and National Security, Committee on Integrated Computational Materials Engineering, National Research Council, The National Academies Press, Washington, DC, USA, 2008.

[29] Z. Lu and Y. Liu, "Small time scale fatigue crack growth analysis," International Journal of Fatigue, vol. 32, no. 8, pp. 1306-1321, 2010. 
[30] W. Zhang and Y. Liu, "Investigation of incremental fatigue crack growth mechanisms using in situ SEM testing," International Journal of Fatigue. In press.

[31] S. Pommier and M. Risbet, "Time derivative equations for mode I fatigue crack growth in metals," International Journal of Fatigue, vol. 27, no. 10-12, pp. 1297-1306, 2005.

[32] P. J. Rabier, "Some remarks on damage theory," International Journal of Engineering Science, vol. 27, no. 1, pp. 29-54, 1989.

[33] W. June and C. L. Chow, "Subcritical crack growth in ductile fracture with continuum damage mechanics," Engineering Fracture Mechanics, vol. 33, no. 2, pp. 309-317, 1989.

[34] J. P. A. Pereira, Generalized finite element methods for threedimensional crack growth simulations, Ph.D. thesis, University of Illinois, Urbana, Ill, USA, 2010.

[35] J. T. Oden, T. Belytschko, I. Babuska, and T. J. R. Hughes, "Research directions in computational mechanics," Computer Methods in Applied Mechanics and Engineering, vol. 192, no. 7-8, pp. 913-922, 2003.

[36] H. T. Banks, "Remarks on uncertainty assessment and management in modeling and computation," Mathematical and Computer Modelling, vol. 33, no. 1-3, pp. 39-47, 2001.

[37] G. Stefanou, "The stochastic finite element method: past, present and future," Computer Methods in Applied Mechanics and Engineering, vol. 198, no. 9-12, pp. 1031-1051, 2009.

[38] P. B. Nair and A. J. Keane, "Stochastic reduced basis methods," AIAA Journal, vol. 40, no. 8, pp. 1653-1664, 2002.

[39] S. Acharjee and N. Zabaras, "A non-intrusive stochastic Galerkin approach for modeling uncertainty propagation in deformation processes," Computers and Structures, vol. 85, no. 5-6, pp. 244-254, 2007.

[40] X. F. Xu, "A multiscale stochastic finite element method on elliptic problems involving uncertainties," Computer Methods in Applied Mechanics and Engineering, vol. 196, no. 25-28, pp. 2723-2736, 2007.

[41] A. Nouy, A. Clément, F. Schoefs, and N. Moës, "An extended stochastic finite element method for solving stochastic partial differential equations on random domains," Computer Methods in Applied Mechanics and Engineering, vol. 197, no. 51-52, pp. 4663-4682, 2008.

[42] D. Post, "The Opportunities and Challenges for Computational Science and Engineering," Julich, Germany, 2007.

[43] S. A. Morton, D. R. McDaniel, D. R. Sears, B. Tillman, and T. R. Tuckey, "Kestrel—a fixed wing virtual aircraft product of the CREATE program," in Proceedings of the 47th AIAA Aerospace Sciences Meeting, Orlando, Fla, USA, January 2009, AIAA 2009-338.

[44] P. C. Chen, D. H. Baldelli, and J. Zeng, "Dynamic flight simulation (DFS) tool for nonlinear flight dynamic simulation including aeroelastic effects," in Proceedings of the AIAA Atmospheric Flight Mechanics Conference and Exhibit, Honolulu, Hawaii, USA, 2008, AIAA 2008-6376.

[45] E. H. Glaessgen, E. Saether, S. W. Smith, and J. D. Hochhalter, "Modeling and characterization of damage processes in metallic materials," in Proceedings of the 52nd AIAA/ASME/ASCE/AHS/ASC Structures, Structural Dynamics and Materials Conference, Denver, Colo, USA, 2011, AIAA 2011-2177.

[46] D. Kinard, "The Digital Thread-Key to F-35 Joint Strike Fighter Affordability," Aerospace Manufacturing and Design, 2010, http://www.onlineamd.com/amd-080910-f-35-joint-strikefighter-digital-thread.aspx. 



Submit your manuscripts at

http://www.hindawi.com
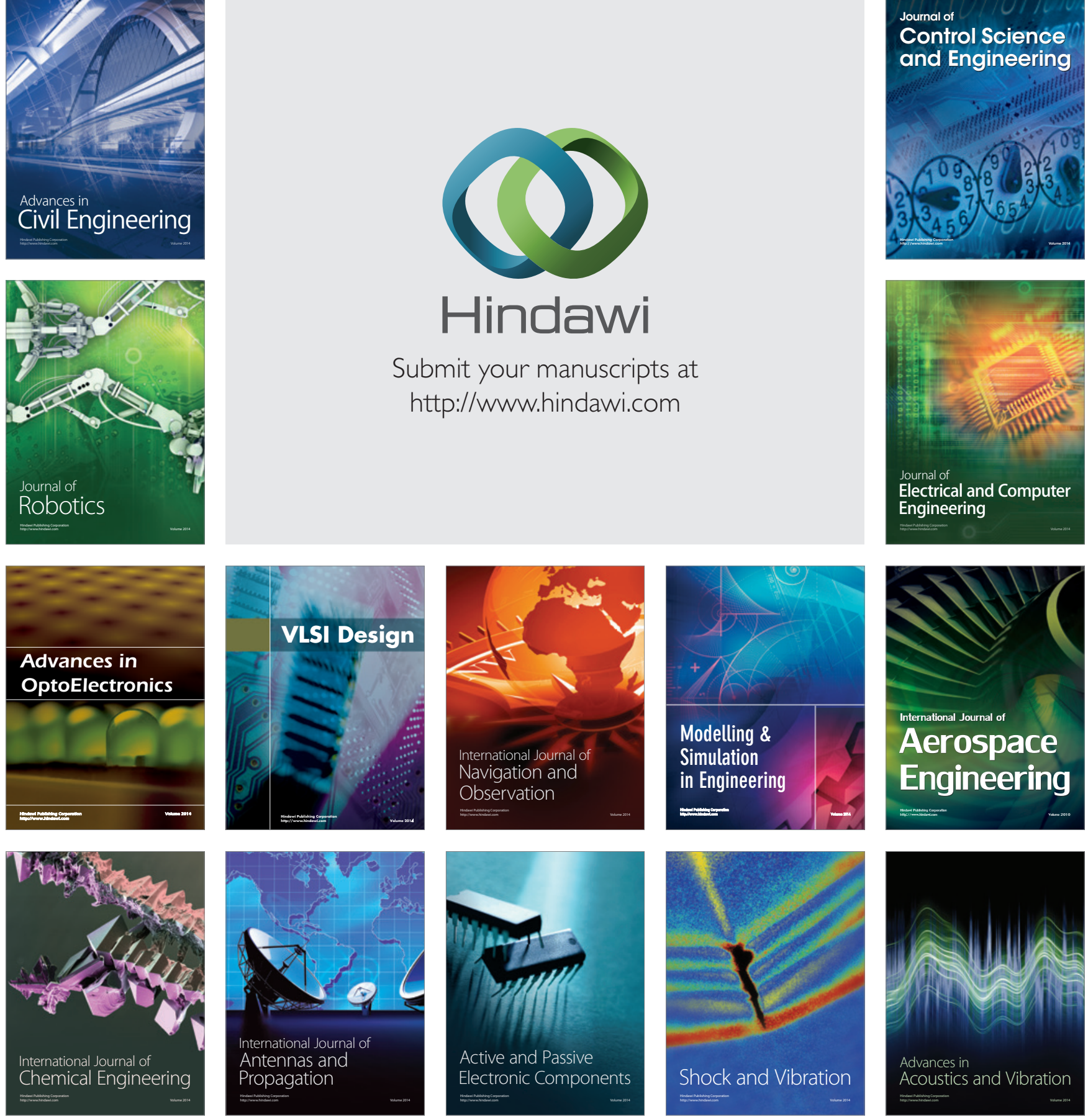